\title{
Estimation of Curvature and Tangent Direction by Median Filtered Differencing
}

\author{
J. Matas, Z. Shao, and J. Kittler \\ University of Surrey, Guildford, Surrey GU2 5XH, United Kingdom
}

\begin{abstract}
We present a new method, median filtered differencing, for estimation of tangent direction and curvature of digitised curves. On three synthetic examples and two images we show the algorithm performs successfully on both straight and curved segments even in the neighbourhood of discontinuities.
\end{abstract}

\section{Introduction}

Curvature estimation is closely linked to a number of problems studied in connection with object recognition, eg. curve partitioning [3], corner detection [10] or extraction of salient points [15]. It is therefore not surprising that a number of curvature estimation methods have been proposed in literature; see [18] [4] [8] for recent surveys.

Worring [17] recognises three classes of approaches to curvature estimation: orientation based [13] [1] [2], path based [9] [11] [10] [14] and osculating circle based [16]. The classification is based on disparate definitions of discrete curvature. The formulations widely differ, but the central underlying assumption remains similar: the discrete curve, typically defined as a sequence of 8-connected pixels, represents a set of samples of the original continuous curve corrupted by uncorrelated gaussian noise. In a subsequent step the least squares machinery is invoked to fit a spline [11], low order polynomial [7] or circle[16]. Kalman filtering [14] requires a similar assumption.

In our opinion sampling of a continuous curve followed by corruption by additive independent gaussian noise is a very poor model of digital curve formation. First, the very fact that edge detection, boundary tracking etc. produces a connected sequence shows the dependence of noise at neighbouring pixels. Rasterisation noise, which can be defined as the distance from a smooth continuous curve to the the nearest point on a discrete grid, is strongly spatially correlated (see fig.4b) and not at all gaussian. The poor match between standard assumption and the actual process of formation and detection of discrete curves might be one of the reasons why Flynn [4] had to conclude that the curvature estimation methods he studied give reasonable results for images with noiseless, real-valued measurements, but none of the five methods gave good estimates after truncation even in the absence of additive noise. Another significant problem related to least square approaches stems from the lack of robustness. Usually, as in images depicted in figures $4 \mathrm{a}$ and $7 \mathrm{a}$, the analysed curve has only piecewise smooth derivatives. Fits computed in the neighbourhood of a discontinuity are distorted as they are based on data belonging to two different smooth segments.

In the paper we propose a new method for estimating tangent direction and curvature based on two assumptions: 1 . errors due to noise and rasterisation are symmetrically 
distributed and 2. at any location along a curve, at least $50 \%$ of neighbouring points belong to the same smooth segment. These assumptions lead naturally to the median filtered differencing method described in section 2. Experiments on curvature and tangent direction estimation on curves linear and curved segments separated by discontinuities in orientation and curvature are reported in section 3. Results are summarised in section 4.

\section{Median filtered differencing}

In the proposed method curvature estimates are obtained by two differencing steps. First, to estimate the tangent direction, median filtered differencing is applied to a sequence of points representing the digital curve. Next, the same computation is performed on the sequence of tangent direction estimates. Will present the algorithm in the form used for tangent direction estimation because of its intuitive geometric interpretation.

Algorithm 1: Median filtered differencing

1. Let $p_{i}, i=1, \ldots, N$ be a set of 8-connected pixel locations. At each $p_{i}$ define a set of $2 M$ difference vectors $d_{i, i+j}, j=-M, \ldots,-1,1, \ldots, M$, such that

$$
d_{i, i+j}=\left\{\begin{array}{l}
p_{i+j}-p_{i} j=1, \ldots, M \\
p_{i}-p_{i+j} j=-1, \ldots,-M
\end{array}\right.
$$

2. Represent $d_{i, .}$ in polar coordinates. Sort $d_{i,}$ according to the polar angle $\theta$.

3. Let $\theta_{i}$ be the $i-t h$ angle in the sorted sequence. The direction of tangent $d_{i r_{t}}$ is estimated as a median of the $2 M$ angles:

$$
\operatorname{dir}_{t}=\left(\theta_{M}+\theta_{M+1}\right) / 2
$$

Examples of the application of algorithm 1 shown in figures 1-3 represent prototypical situations. Figure 1 depicts a noisy rasterised straight segment. The difference vectors $d_{i,}$, are shown in fig. 1a, sorted and translated in fig.1b. Vectors marked ' $2 x^{\prime}$ ' appear twice. In this example, $M$ equals four. The median angle, ie. the estimated tangent direction, of the eight difference vectors is denoted $d i r_{t}$. Note that for a straight line, the differences from points farthest from $p_{i}$ are in the center of the distribution because they are least influenced by rasterisation noise.

Figure 2 demonstrates behaviour of alg. 1 near a discontinuity in orientation. The distribution of $d_{i,}$. consists of two components: the directions belonging to the segment with $p_{i}$ and outlier directions from the other side of the discontinuity. As $p_{i}$ approaches the discontinuity, the proportion of outliers will grow, but will remain just under $50 \%$. The median is therefore not grossly influenced [5]. Unlike methods based on smoothing the median differencing performs well near corners, regardless of the size $M$ of the neighbourhood used to obtain the estimate. 


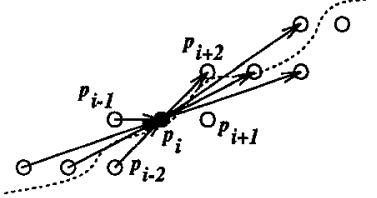

(a)

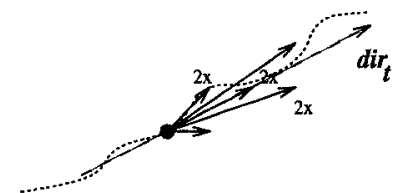

(b)

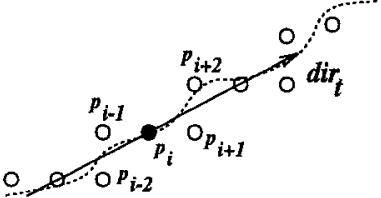

(c)

Fig. 1.: Median filtered differencing on noisy rasterised straight line. (a) Sequence of points around $p_{i}$ and the $2 M$ difference vectors, $M=4$ (b) Difference vectors translated to $p_{i}$. (c) The estimated tangent direction $\operatorname{dir}_{t}$ superimposed on the original curve.

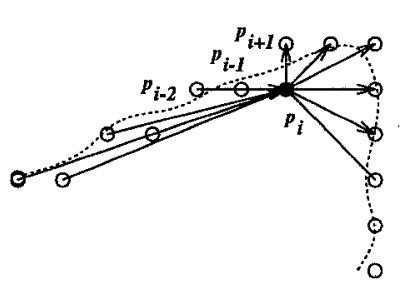

(a)

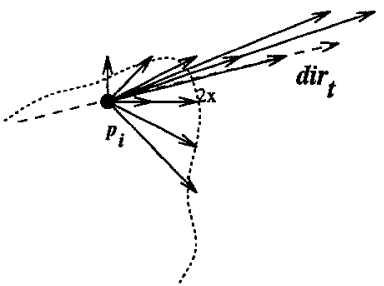

(b)

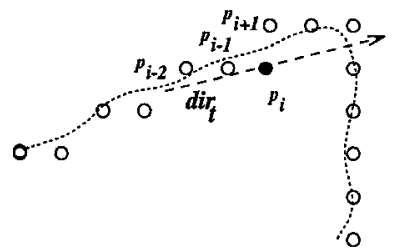

(c)

Fig. 2.: Median filtered differencing. (a) Sequence of points around $p_{i}$ and the $2 M$ difference vectors, $M=6$ (b) (c) see fig. 1

Figure 3 show performance of alg. 1 on a general smooth curve. In this case difference vectors far from $p_{i}$ are strongly biased, vectors from immediate neighbourhood of $p_{i}$ are significantly influenced by discretisation. The median is most likely drawn from difference vectors with average distance from $p_{i}$ (see figs. $3 \mathrm{a}-\mathrm{c}$ ). To summarise, we can conclude that the algorithm behaved intuitively correctly in all three cases. On a straight segment it estimated the tangent direction from points as far apart as possible. Near a breakpoint, outliers were rejected. On a smooth curve with non-zero curvature the algorithm finds a compromise between systematic bias and errors due to noise.

The same algorithm was used for curvature estimation with $d_{i, i+j}$ replaced with $(\delta \theta, j)$. The algorithm effectively selects a median slope of the tangent direction as function of arc length.

As implemented the efficiency of the method is $O(N M \log M)$, ie. it is linear in the number of points at which estimates are sought. The sorting of directions is responsible

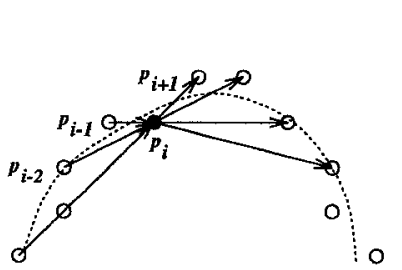

(a)

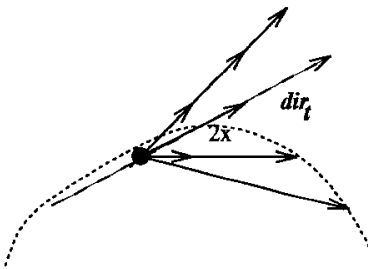

(b)

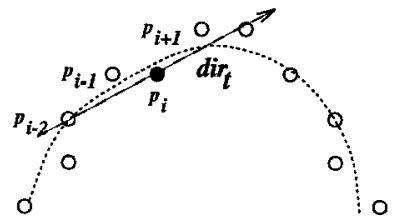

(c)

Fig. 3.: Median filtered differencing on a general smooth curve. (a) Sequence of points around $p_{i}$ and the $2 M$ difference vectors, $M=4$. (b)(c) see fig. 1 


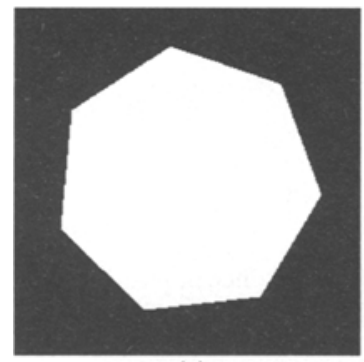

(a)

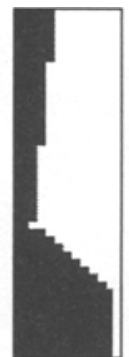

(b)

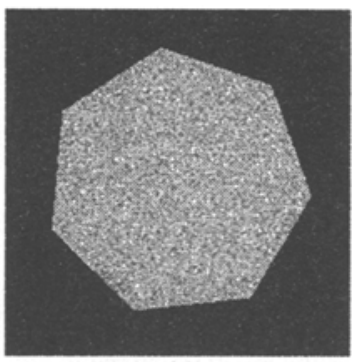

(c)

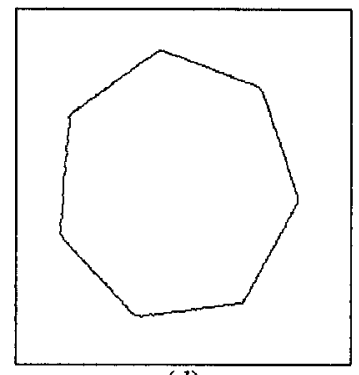

(d)

Fig. 4.: Heptagon. (a) Rasterised, noiseless. (b) Magnification of the area around the leftmost corner. Notice the artifact at the vertex. (c) Gaussian noise added; foreground $N(200,30)$, background $\mathrm{N}(50,30)$. (d) noisy boundary output by Canny edge detector.

for the $M \log M$ term. It is known that median can be found in almost linear time [12]. For the neighbourhood sizes used in our experiments with $M$ between five and fifteen we decided that the improved asymptotic complexity did not justify the effort to implement the more sophisticated algorithm.

\section{Experiments}

Due to lack of space we will describe only experiments on two 2D shapes. The heptagon depicted in fig. 4a, was chosen because it demonstrates performance of our algorithm on linear segments with different directions with respect to the discrete grid. The silhouette of scissors shown in fig. 7 comprises a number of smooth segments as well as a number of discontinuities.

Figures 5 and 6 summarise performance of median filtered differencing on the heptagonal shape. Fig. 5a shows the angle estimate computed on the boundary of the noiseless rasterised image 4a. The results is close to the 'ideal' step function showing that the algorithm is a capable of filtering the highly correlate rasterization noise. In a

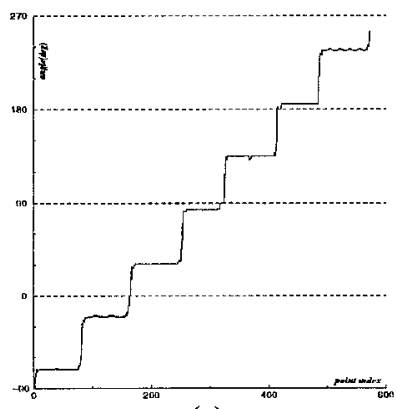

(a)

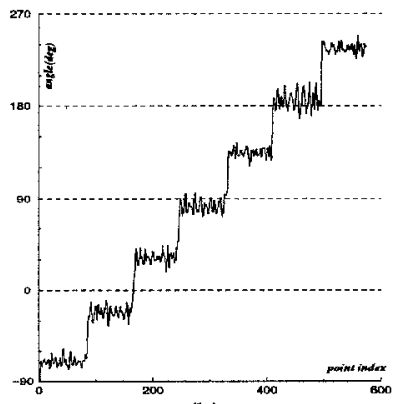

(b)

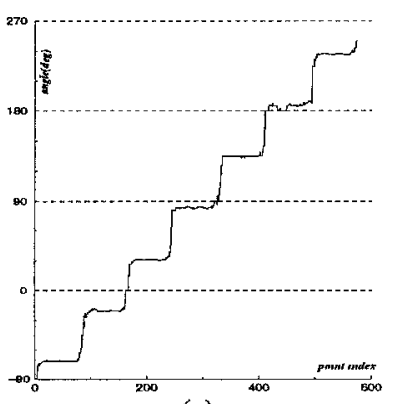

(c)

Fig. 5.: Heptagon. Tangent angle estimation. (a) Median differencing of noiseless rasterised image 4(a), $M=13$. (b) Canny edge detection, gradient direction (c) median differencing on edge string $4 \mathrm{~d}, M=13$ 


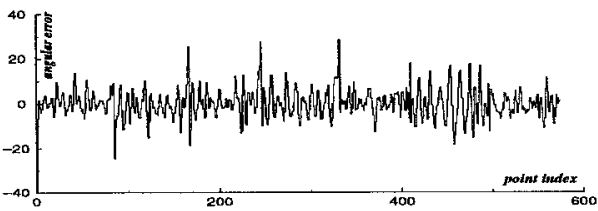

(a)

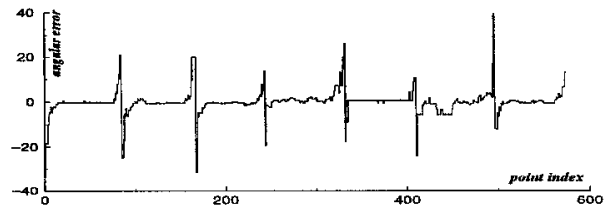

(b)

Fig. 6.: Heptagon. Error of tangent angle estimation for the Canny edge detector(a)(see fig. 5b) and the median filtered difference on the edge string (see fig. 5c).

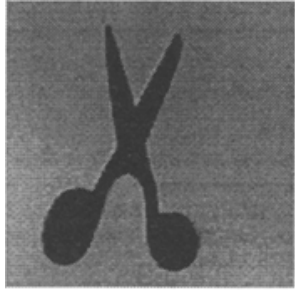

(a)

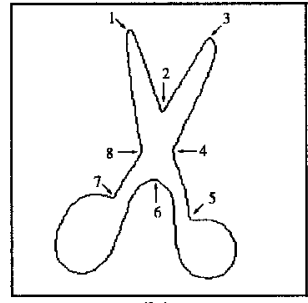

(b)

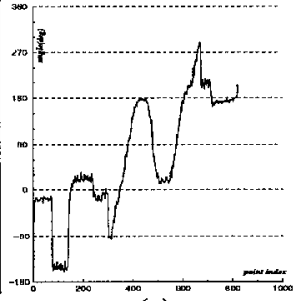

(c)

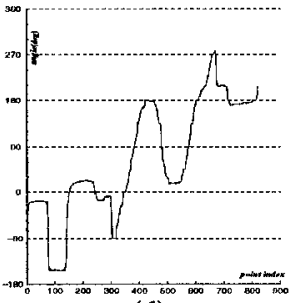

(d)

Fig. 7.: Scissors. (a) Original image. (b) Boundary output by Canny edge detector. (c) Canny edge detector, gradient direction. (d) Tangent angle direction estimated by median differencing, $M=15$

following experiment, gaussian noise was added to the heptagon image (fig. 4c). Next, we run Canny edge detector to obtain boundary of the heptagon (fig. 4d). Edge direction is a part of the edge detector output (fig. 4b). We applied algorithm 1 to the edge string. Figures $5 \mathrm{~b}-\mathrm{c}$ and $6 \mathrm{a}-\mathrm{b}$ compare the results. On the straight section, the standard deviation of the error in angle estimate (from the known ground truth) is reduced by a factor of 10 . To achieve similar results by linear filtering, significant smoothing would take place at corners. When interpreting the comparatively large errors of median differencing near the corners we have to bear in mind the corners were rounded off by edge detection, not by smoothing of the estimation process.

The first part of the 'scissors' experiment, summarised in figure 7, shows that the median differencing produces reliable estimates of the tangent direction on a complex contour. Comparing figs. $7 \mathrm{c}$ and $7 \mathrm{~d}$ it is clear that the noise reduction is not accompa-

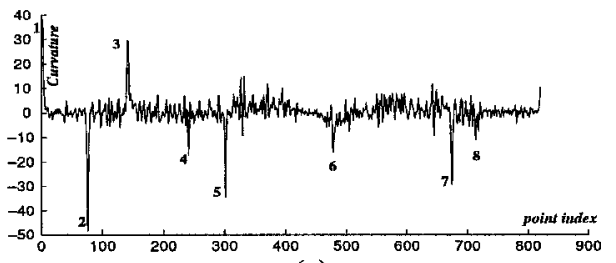

(a)

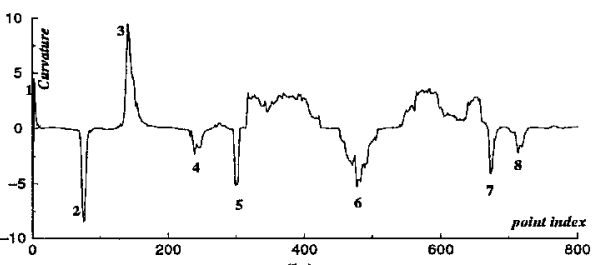

(b)

Fig. 8.: Scissors. Curvature estimation. (b) Differentiated gradient direction (fig. 7c). (c) Median filtered differencing of angle estimate (fig. 7d). Numbers mark points of maximum convexity and concavity (above a threshold). median 
nied by smoothing at discontinuities; steps and peaks remain sharp. Results of curvature computation are presented in fig. 8. The contrast in smoothness of figs. $8 \mathrm{a}$ and $8 \mathrm{~b}$ is apparent. Selection of points of maximum convexity and concavity is easily accomplished by non-maximum suppression.

\section{Conclusions}

We have presented a new method, median filtered differencing, for estimation of tangent direction and curvature. On three synthetic examples and two images we show the algorithm performs successfully on both straight and curved segments even in the neighbourhood of discontinuities.

\section{References}

1. I.M. Anderson and J.C. Bezdek. Curvature and tangential deflection of discrete arcs: a theory based on the commutator of scatter matrix pairs and its application to vertex detection in planar shape data,. IEEE Transactionson Pattern Analysis and Machine Intelligence, 6:27-40, 1984.

2. H. Asada and M. Brady. The curvatureprimal sketch. IEEE Transactions on Pattern Analysis and MachineIntelligence, 8:2-14, 1986

3. Martin A. Fishler and Helen C. Wolf. Locating perceptually salient points on planar curves. IEEE Transactions on Pattern Analysis and Machine Intelligence, 16:113-128, February 1994.

4. Patrick J. Flynn and Anil K. Jain. On reliable curvature estimation. In CVPR'88 (IEEE Computer Society Conference on Computer Vision and Pattern Recognition, Ann Arbor, MI, June 5-9, 1988), pages 110-117, Washington, DC., June 1988. Computer Society Press.

5. F. R. Hampel, E. M. Ronchetti, P.J. Rouseseeuw, and W.A. Stahel. Robust Statistics. John Wiley, 1986.

6. 11th IAPR International Conference on Pattem Recognition (The Hague, The Netherlands, August 30-September 3, 1992), Washington, DC, 1992. IEEE Computer Society Press.

7. C. Lee, R. Haralick, and K. Deguchi. Estimation of curvature from sampled noisy data. In CVPR'93 (IEEE Computer Society Conference on Computer Vision and Pattern Recognition, New York City, NY, June 15-17, 1993), pages 536-541, Washington, DC., Jun 1993. Computer Society Press.

8. R. Legault and C.Y. Suen. A comparison of methods of extracting curvature features. In ICPR1992 [6], pages 134-138.

9. D.G. Lowe. Organization of smooth image curves at multiple scales. In Second International Conference on Computer Vision (Tampa, FL, December 5-8, 1988), pages 558-567, Washington, DC., 1988. Computer Society Press.

10. G. Medioni and Y. Yasumoto. Corner detection and curve representation using B-splines. Computer Vision. Graphics and Image Processing, 39:267-278, 1987.

11. T. Pavlidis. Application of splines to shape description. In Visual Forms - Analysis and Recognition, Proceedings of International Workshop on Visual Form, pages 431-441, 1991.

12. W.H. Press, B.P. Flannery, S.A. Teukolsky, and W.T. Vetterling. Numerical Recipes in C. Cambridge University Press, 1988.

13. A. Rosenfeld and E. Johnston. Angle detection in digital curves. IEEE Trans. Computers, C-24:875-878, September 1973.

14. P.T. Sander. Estimating curvature by kalman filters. In Visual Forms - Analysis and Recognition, Proceedings of International Workshop on Visual Form, pages 469-477, 1991.

15. Zhimin Shao and Josef Kittler. Estimating angles and curvature features in grey scale images. In E. Hancock, editor, British Machine Vision Conference, pages 115-124. BMVA Press, 1994.

16. S.M. Thomas and Y.T. Chan. A simple approach for the estimation of circular arc center and its radius. Computer Vision, Graphics and Image Processing, 45:362-370, 1989.

17. M. Worring and A. W. M. Smeulders. The accuracy and precision of curvature estimation methods. In ICPR1992 [6], pages 139-142.

18. M. Worring and A. W. M. Smeulders. Digital curvature estimation. CVGIP: Image Understanding, 58:366-382, November 1993 